\title{
Neurocisticercosis racemosa
}

\section{Racemose neurocysticercosis}

\author{
EnverVantroi Palacios-Ordóñez,' Ysabel Lozano-Rodas ${ }^{1,2}$ \\ y Fiorella Carolina Portilla-Paredes ${ }^{3}$
}

Palacios-Ordóñez EV, Lozano-Rodas Y, Portilla-Paredes FC. Neurocisticercosis racemosa. Rev Soc Peru Med Interna. 2019;32(I):29-33.

\begin{abstract}
RESUMEN
Mujer de 44 años de edad que ingresó por emergencia con cefalea holocraneana, progresiva e intensa, vómitos explosivos y hemiparesia derecha progresiva, asociados con múltiples caídas. Las imgenes evidenciaron formaciones quísticas conglomeradas en el valle silviano izquierdo y en parte superior de la cisterna pretroncal no captadoras de contraste, planteando el diagnóstico de neurocisticercosis racemosa.

Palabras Clave. Neurocisticercosis, racemosa, Taenia solium.
\end{abstract}

\section{ABSTRACT}

A 44-year-old woman who entered to emergency room presenting holocraneal, progressive and intense headache, explosive vomits and progressive right hemiparesis associated with multiple falls. The images showed a cystic formations conglomerate in the left silvian valley and at top of pretroncal cistern and not enhanced by contrast, considering the diagnosis of racemose neurocysticercosis.

Key words: neurocysticercosis, racemose, Taenia solium.

\section{INTRODUCCIÓN}

La neurocisticercosis (NCC) es una de las infecciones helmínticas más comunes del sistema nervioso central causada por la etapa larvaria de la Taenia solium, y es una de las causas más comunes de epilepsia secundaria en los países en vías de desarrollo. Cerca de 50 millones de personas en el mundo están infestadas por el complejo teniasis-cisticercosis y 50000 de ellas mueren cada año por esta causa. ${ }^{1-3}$

El cisticerco puede localizarse a nivel parenquimal y asociarse a cuadros convulsivos, o a nivel extraparenquimal y ocasionar hipertensión endocraneana.

I. Médico internista. Hospital María Auxiliadora, Lima.

2. Médico internista. Hospital María Auxiliadora. Facultad de Medicina, Universidad Científica del Sur, Lima

3. Médico residente. Hospital María Auxiliadora, Lima
La NCC extraparenquimal es poco frecuente, incluye quistes subaracnoideos o intraventriculares. Los quistes subaracnoideos pueden alcanzar gran tamaño y tienden a ubicarse en la base del cerebro o en la cisura de Silvio; en tanto que los intraventriculares provocan elevación transitoria o persistente de la presión intracraneal secundaria al bloqueo del drenaje del líquido cefalorraquídeo. ${ }^{4,5}$

La clínica es pleomórfica. Se presentan desde formas asintomáticas hasta síntomas graves, que dependen de la localización del cisticerco. En revisiones sistemáticas, ${ }^{6,7}$ se encontró lo siguiente: convulsiones $(79 \%)$, cefalea (40\%), déficit neurológico focal $(16 \%)$ y signos de hipertensión intracraneal (12\%); y, en menos del $10 \%$ de los casos, se presentaron ataxia, alteraciones visuales, parálisis de nervios craneales, síntomas meníngeos y alteraciones del estado mental. La presentación extraparenquimal suele tener un comportamiento agresivo dada su gran predisposición a producir complicaciones. Si las vesículas se alojan en cisternas amplias, como la silviana, pueden crecer libremente hasta adquirir un tamaño gigante (> $50 \mathrm{~mm}$ ), dando lugar a la denominada forma racemosa. ${ }^{8}$ A continuación se presenta el caso de una paciente que presentó NCC racemosa asociado a hipertensión endocraneana $\mathrm{y}$ hemiparesia derecha. 


\section{REPORTE DEL CASO}

Mujer de 44 años, natural y procedente de Yurimaguas, San Martín, ama de casa, primaria incompleta, sin antecedentes patológicos. Ingresó con tiempo de enfermedad de un mes, inicio insidioso, curso progresivo, presentaba cefalea holocraneana tipo opresiva de cortos periodos de duración. Tres semanas antes de su ingreso la cefalea se incrementó y se asoció a vómitos explosivos y disminución progresiva de la fuerza muscular del hemicuerpo derecho, que cursó con múltiples caídas.

\section{Examen físico}

Funciones vitales estables, regular estado general.

Piel tibia, mucosas orales húmedas, orofaringe no congestiva.

Aparato respiratorio: murmullo vesicular normal en ambos campos pulmonares.

Aparato cardiovascular: ruidos cardíacos rítmicos, no soplos.

Abdomen: blando, depresible, ruidos hidroaéreos presentes, no visceromegalia, no masas palpables.

Sistema nervioso central: despierta, lúcida, orientada en tiempo, espacio y persona, colabora con el examen; pares craneales: oculomotores normales, motilidad y sensibilidad del rostro conservados; motor: hemiparesia derecha proporcional $3+/ 5$; sensibilidad: disminución de la sensibilidad termoalgésica del hemicuerpo derecho, no compromiso de sensibilidad profunda. Reflejos miotáticos: hemicuerpo derecho 5+, hemicuerpo izquierdo 3+. Babinski derecho presente. No signos meníngeos. Fondo de ojo sin particularidades.

\section{Exámenes auxiliares}

Hemograma: leucocitos, 12 900/mm ${ }^{3}$ (abastonados $1 \%$, segmentados $83 \%$, linfocitos $8 \%$, monocitos $8 \%$, eosinófilos $0 \%$, basófilos $0 \%$ ); plaquetas 254000 / $\mathrm{mm}^{3}$. Hemoglobina $12,9 \mathrm{~g} / \mathrm{dL}$.

Volumen corpuscular medio (VCM), 88; hemoglobina corpuscular media (HCM), 29,3; hematócrito, $39 \%$.

Creatinina, 0,61 mg/dL; glucosa, $117 \mathrm{mg} / \mathrm{dL}$; urea, $32 \mathrm{mg} / \mathrm{dL}$.

Electrólitos: $\mathrm{K}^{+} 3,9 \mathrm{mEq} / \mathrm{L} ; \mathrm{Na}^{+} 137 \mathrm{mEq} / \mathrm{L} ; \mathrm{Ca}^{2+}$ 1,09; $\mathrm{HCO}_{3} 24,5 \mathrm{mEq} / \mathrm{L}$; lactato $2,4 \mathrm{mmol} / \mathrm{L}$.

Tomografía cerebral sin contraste: múltiples imágenes quísticas a nivel temporoparietal izquierdo, desviando la línea media y obturando el ventrículo lateral del mismo lado (Figura 1).

Resonancia magnética nuclear con contraste (Figura 2): imágenes hipointensas en $\mathrm{T} 1$ e hiperintesas en $\mathrm{T} 2$ compatibles con formaciones quísticas conglomeradas en el valle silviano izquierdo, que comprimen el tercer ventrículo y la parte superior de la cisterna pretroncal, no captadoras de contraste.

Con las imágenes compatibles con NCC racemosa, se solicitó examen de Inmunoblot para cisticercosis. Se detectó anticuerpos específicos contra larva o cisticerco de Taenia solium (presencia de 7 bandas de diagnóstico).

\section{Tratamiento}

La paciente fue transferida a sala de neurocirugía, donde se realizó craneotomía frontotemporal y extracción de quistes. Posteriormente, se le realizó control, para el inicio de tratamiento antiparasitario.

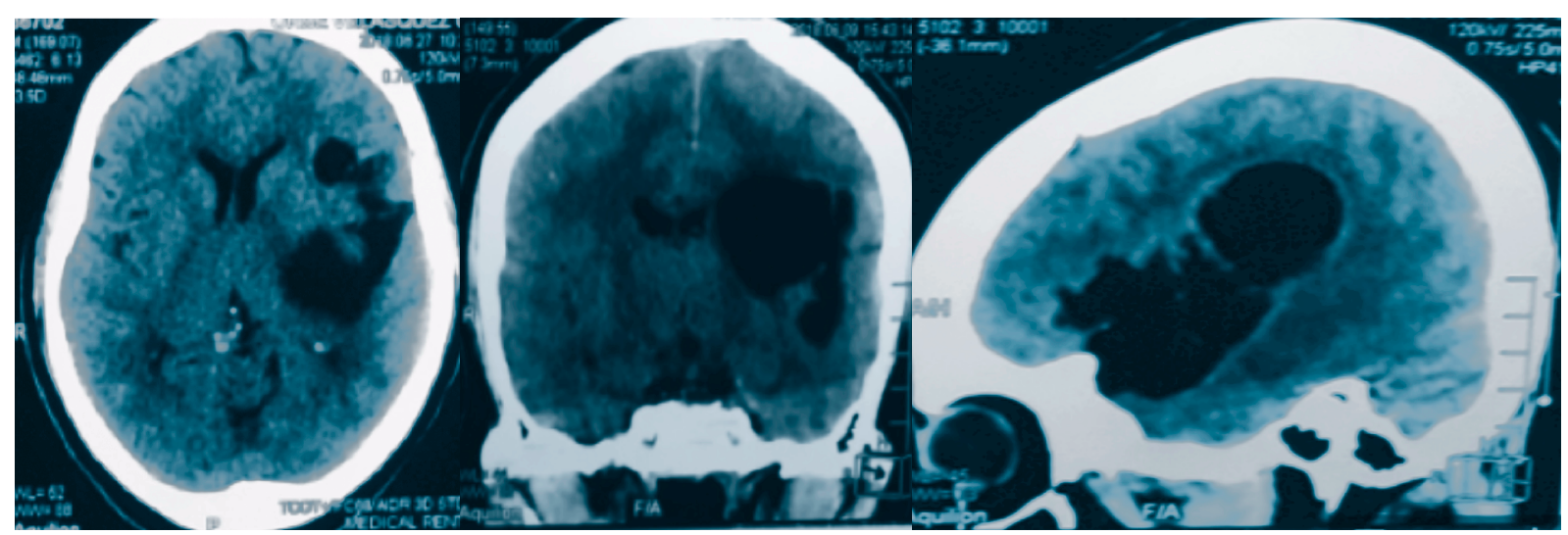

Figura I.Tomografía cerebral sin contraste en tres cortes.A) Axial. B) Coronal. C) Sagital. 


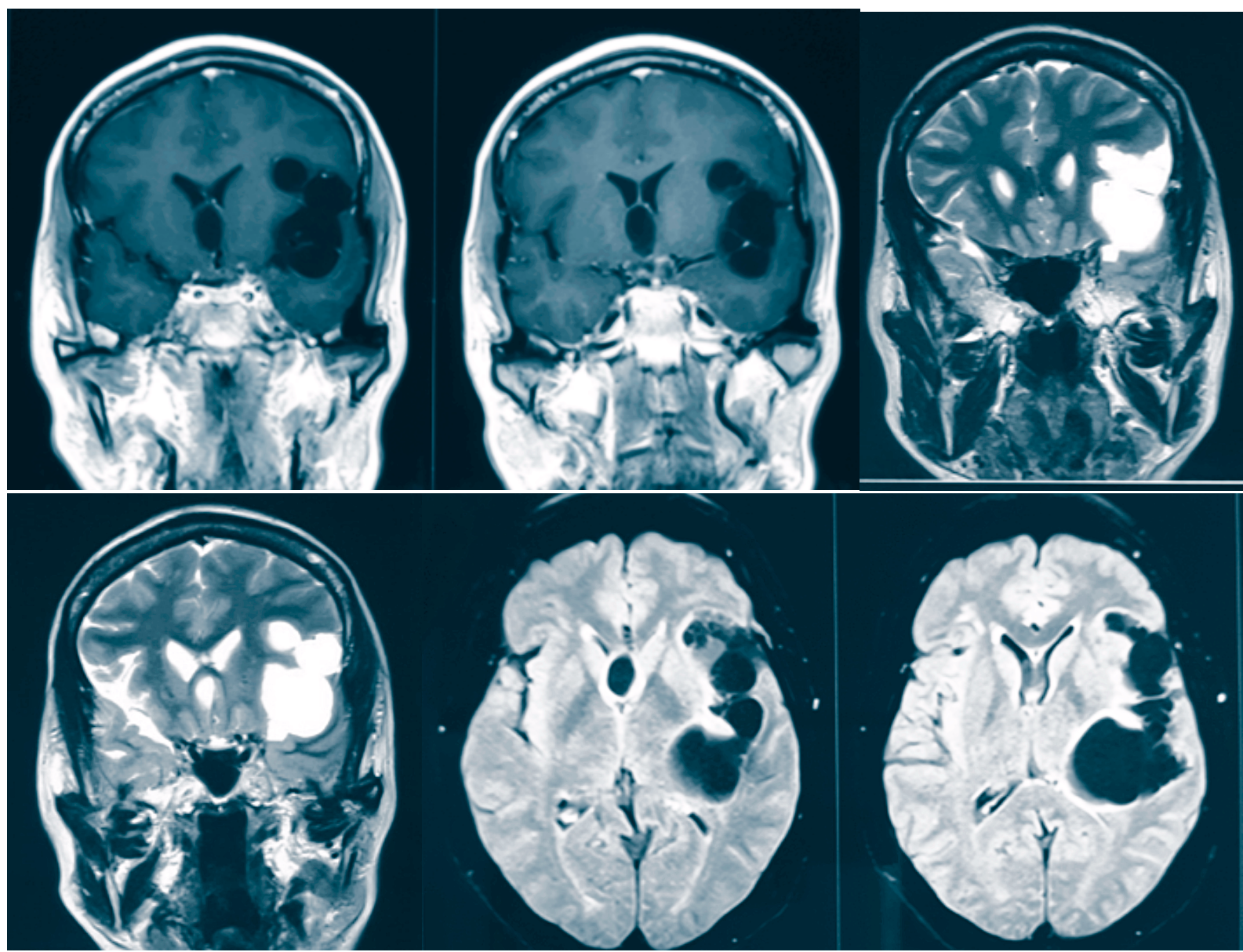

Figura 2. Resonancia magnética: TI múltiples imágenes quísticas conglomeradas hipointensas que se tornan hiperintensas en T2. Comprime el tercer ventrículo y la parte superior de la cisterna pretroncal. Evidencia de complejo vesicular membranoso por proliferación continua de membranas del parásito.

\section{DISCUSIÓN}

La cisticercosis es una zoonosis causada por la etapa larval enquistada de T. solium. Puede afectar cualquier órgano del cuerpo, pero el parénquima cerebral es el lugar más afectado del sistema nervioso central $(60 \%$ $90 \%$ ), y constituye una de las causas más comunes de epilepsia secundaria en países en vías de desarrollo. Análisis recientes calculan que el $29 \%$ de la epilepsia en regiones endémicas en todo el mundo es causada por NCC, un valor que se aproxima mucho a las estimaciones de América Latina. Cerca de 50 millones de personas en el mundo están infestadas por el complejo teniasiscisticercosis y 50000 de ellas mueren cada año por esta causa. $^{1-3}$

Las comunidades rurales presentan una alta prevalencia de teniasis y cisticercosis, alcanzando $17 \%$ y $75 \%$ en humanos y porcinos, respectivamente. Ello se ve favorecido por factores como pobreza, escasa educación sanitaria, falta de higiene, ausencia de letrinas, eliminación de excretas y residuos sólidos al aire libre, consumo de agua no potable, crianza clandestina de cerdos, manejo inadecuado de las carcasas y el consumo de esta carne sin inspección veterinaria, ${ }^{5}$ condiciones que favorecen la alta prevalencia de esta zoonosis.

Después de la ingestión, las oncósferas son transportadas por el torrente sanguíneo a varios órganos y se alojan en los vasos sanguíneos pequeños donde pueden o no convertirse en quistes viables $(2$ a 3 meses). La distribución de los quistes generalmente sigue la distribución de la sangre en el cerebro. Los quistes parenquimatosos son más comunes y en las infecciones graves frecuentemente se localizan en las áreas de la cuenca hidrográfica entre la materia blanca y gris. Por qué y cómo se desarrollan algunos quistes en subaracnoideos o racemosos grandes se desconoce, pero puede ocurrir después de alguna forma de degeneración y pérdida de la inhibición del crecimiento. 
Así, en la NCC racemosa, el parásito alcanza por vía hematógena las cisternas basales, espacios subaracnoideos y meninges, desencadenando una intensa reacción inflamatoria perilesional. Esta reacción inflamatoria puede afectar las leptomeninges en la base del cráneo, extenderse hasta el agujero magno y producir una leptomeningitis basilar que engloba nervios y arterias craneales. También se afectan los agujeros de Luschka y Magendie con hidrocefalia. ${ }^{4}$ La forma meníngea se presenta con elevación de la presión intracraneal debido a varias causas: meningitis generalizada y adherencias que resultan en obstrucción del flujo de líquido cefalorraquídeo (LCR) e hidrocefalia. Puede causar posteriormente vasculitis y atrapamiento de los nervios craneales en la inflamación, dando como resultado un déficit neurológico focal. El atrapamiento de los nervios oculomotores puede causar parálisis muscular extraocular y diplopía. El nervio óptico y el quiasma óptico pueden encerrarse dentro del exudado, causando defectos de visión y campo visual. ${ }^{2}$

En la forma intraventricular o subaracnoideas (cisternas), las oncósferas alcanzan los ventrículos a través del plexo coroideo. Estos parásitos ocluyen la vía del LCR causando episodios agudos de ventriculomegalia y un efecto de masa. En particular, la forma subaracnoidea puede producir enfermedad cerebrovascular, incluidos infarto cerebral, ataques isquémicos transitorios y hemorragia cerebral. El mecanismo subyacente más común es el de inducción de arteritis encontrados en el $54 \%$ de 28 pacientes. Las arterias cerebral media y cerebral posterior son los vasos cerebrales más comúnmente involucrados. ${ }^{2}$ Por lo tanto, la mayor parte de la fisiopatología resulta directa o indirectamente de la localización e inflamación aguda o crónica debida a quistes degenerados, membranas, restos de quistes y antígeno residual. Controlar la inflamación es una piedra angular para limitar la morbilidad y la mortalidad. Procesos menos frecuentes son el efecto de masa y la obstrucción mecánica. Muchos pacientes presentan enfermedad intraparenquimal y extraparenquimal complicada, múltiples ubicaciones en diferentes etapas de evolución y grados variables de inflamación asociados con cada lesión o área de afectación. La manifestación más grave será la que dicta el enfoque inicial. ${ }^{3}$

La clínica usual de presentación de la NCC en nuestro medio son las convulsiones, presentándose en el tipo parenquimatosa usualmente calcificada, por lo que no suele requerir un tratamiento antiparasitario más sí fármacos antiepilépticos. Por tanto, podemos decir que la mayoría de pacientes evaluados en los servicios de emergencia ingresan por complicaciones de esta entidad.

La forma intraventricular de NCC requiere intervención pronta y agresiva en vista de su rápida progresión clínica, y a menudo se asocia con un pobre pronóstico. Puede ocurrir junto con la forma parenquimatosa o, más comúnmente, aislada. El cuarto ventrículo es el sitio más común $(53 \%)$, seguido por el tercer ventrículo $(27 \%)$, ventrículo lateral $(11 \%)$ y el acueducto $(9 \%)$. Los quistes ventriculares también pueden inducir el síndrome de Bruns, que se caracteriza por dolor de cabeza episódico, vómitos, papiledema, cuello rigidez y vértigo posicional repentino inducido por movimientos rotatorios de la cabeza, ataques de gota y pérdida de conciencia con una recuperación rápida. ${ }^{3}$

En casos raros, la NCC racemosa puede causar demencia como única característica de presentación en el paciente. Sin embargo, $66 \%$ a 87,5\% de pacientes con NCC han sido reportados tener alteraciones cognitivas, demencia o deterioro cognitivo grave en $12,5 \%$ a $15,6 \%$ de los pacientes. $^{2,3}$

Con respecto al diagnóstico, en el año 2000, un grupo de expertos propuso una serie de criterios diagnósticos basados en cuatro categorías. ${ }^{1}$ En relación al diagnóstico imagenológico, la RM es más sensible que la TC para el diagnóstico de la enfermedad ya que permite identificar un mayor número de lesiones. Las secuencias de RM ponderadas en T1 y T2 identifican el quiste del neurocisticerco, cuya señal en el estadio vesicular es idéntica a la del líquido cefalorraquídeo. En el estadio vesicular-coloidal (muerte del parásito), el contenido proteico del quiste aumenta, y también lo hace su señal en las secuencias básicas de resonancia. En este estadio es posible ver además la formación de una cápsula hipointensa en secuencias ponderadas en T2. De los múltiples hallazgos radiológicos que se pueden encontrar en la NCC, la presencia del escólex dentro de la lesión quística es considerada patognomónica. ${ }^{4}$

El tratamiento de esta patología es complejo, depende de la localización, estadio de desarrollo del parasito y la clínica del paciente, por lo que se debe incidir en la prevención de la parasitosis.

El caso reportado representa una variante inusual de NCC extraparenquimal, ${ }^{2-5}$ debido a la migración 
aberrante de T. solium. La paciente tuvo un mes de evolución con un síndrome motor piramidal focalizando en el hemicuerpo derecho, sin convulsiones ni síntomas visuales. Esta es la presentación usualmente crónica de los quistes subaracnoideos gigantes que comprimen el parénquima cerebral de forma progresiva. ${ }^{6} \mathrm{~A}$ diferencia de lo que reportó Kumar y col., ${ }^{7}$ en 2013, un paciente de 22 años con historia de cefalea y convulsiones con tres meses de evolución sin signos neurológicos focales que más tarde tuvo como hallazgo por RM de lesiones quísticas distribuidos en el parénquima de ambos hemisferios cerebrales. Así mismo, en 2016, Arushi Saini y col. ${ }^{8}$ reportaron un paciente de 12 años con historia de cefalea, vómitos y mareos, sin compromiso visual, convulsiones o déficit motor, que más tarde cursó con compromiso de sensorio, evidenciando en la RM una NCC subaracnoidea e intraventricular. Sánchez Larsen y col. reportaron el caso de una mujer de 58 años con episodios de cefalea de incremento en frecuencia e intensidad por RM, con exploración física tanto sistémica como neurológica normal, incluido el fondo de ojo.

Estos casos llevan a evidenciar que la sintomatología suele ser inespecífica debido a su cronicidad y que su presentación con compromiso motor es inusual, llevándonos a que la forma de NCC racemosa cursa con múltiples formas de presentación que pueden pasar desapercibidas para el clínico, las cuales podrían incluso manifestarse con complicaciones graves. Por lo tanto, debemos contribuir a su estudio en poblaciones de riesgo y dar el tratamiento oportuno.

\section{REFERENCIAS BIBLIOGRÁFICAS}

I. Del Brutto OH, RajshekharV,White JrAC,TsangVC, Nash TE, Takayanagui $\mathrm{OM}$, et al. Proposed diagnostic criteria for neurocysticercosis. Neurology. 2001;57:177-83.

2. Mahale RR. Extraparenchymal (racemose) neurocysticercosis and its mutitude manifestations: A comprehensive review. J Clin Neurol. 20I5; I I(3):203-2 II.

3. Nash TE, Garcia HH. Diagnosis and treatment of neurocysticercosis. Nat Rev Neurol. 20I I;7(I0):584-594.

4. Bickerstaff ER, Cloake PCP, Hughes B, Smith WT. The racemose form of cerebral cysticercosis. Brain. 1952;75(I):I I6.

5. Saavedra H, Gonzales I, Alvarado M, Porras M, Vargas V. Diagnóstico y manejo de la neurocisticercosis en el Perú. Rev Peru Med Exp Salud Pública. 2010;27(4):586-91

6. Carabin H, Ndimubanzi PC, Budke CM, Nguyen H, Qian Y, Cowan LD, et al. Clinical manifestations associated with neurocysticercosis: A systematic review. PLoS Negl Trop Dis. 201 I ;5(5):el I 52.

7. Mahale R, Mehta A, Rangasetty S. Extraparenchymal (racemose) neurocysticercosis and its multitude manifestations: A comprehensive review. J Clin Neurol. 2015; I I:203-II.

8. Sánchez-Larsen A, et al. Neurocisticercosis racemosa subaracnoidea gigante y ventricular: a propósito de un caso. Rev Argent Microbiol. 2015;47(3):20I-205.

9. Sarria Estrada L, Frascheri Verzelli S. Neurocisticercosis. Hallazgos radiológicos. Radiología. 2013;55(2):97-I84.

10. Gemmel M, Matyas Z, Pawlowski Z, et al. Guidelines for surveillance prevention and control of teniasis/cysticercosis. Geneva:World Health Organization; 1983. p. I-207.

II. Fleury A, Carrillo-Mezo R, Flisser A, Sciutto E, Corona T. Subarachnoid basal neurocysticercosis: a focus on the most severe form of the disease. Exp Rev Anti-Infect Ther. 20I I;9(I):I23-I33.

12. Kumar S,Thakur S, JhobtaA,Sood RG. Giant racemose neurocysticercosis with mass effect: Unusual presentation. Ann Indian Acad Neurol. 2013 16:398-9.

13. Saini AG, Vyas S, Singhi P. Racemose neurocysticercosis. J Infect Public Health. 2017;10:884-885.

CORRESPONDENCIA:

envervan33@hotmail.com

FECHA DE RECEPCIÓN: 16 de febrero de 2019.

FECHA DE ACEPTACIÓN: 18 de marzo de 2019. 